\title{
Characterizing Dynamic Pressure in Cycling with Muscle Activation
}

\author{
Shunhua Luo ${ }^{1,2^{*}}$, Jianping Wang ${ }^{1}$, Hui Shi ${ }^{1}$, Yang Yang ${ }^{1}$ \\ ${ }^{1}$ Fashion Institute, Donghua University, Shanghai 200051, China \\ ${ }^{2}$ Fashion institute, Shandong University of Art and Design, Jinan Shandong 250300, China
}

Keywords: Dynamic pressure, Compression shorts, Cycling, Muscle activation

\begin{abstract}
Dynamic pressure plays an important role in the cyclists' performance and comfort. To qualitatively analyze dynamic pressure of male lower limb with compression cycling shorts in continuous cycling motion, a novel approach was developed to characterize dynamic pressure. Four pressure measurement points were selected based on skeletal muscle simulation. It was found that pressure was dynamic during cycling, and the maximum dynamic pressure at four points was produced when pedal was at the top. Pressure gradually non-linear rose with ease allowance reduction. Dynamic pressure significantly associated with muscle activation. The investigation will contribute to theoretical guidance for clothing engineers to improve pressure comfort and motor performance.
\end{abstract}

\section{Introduction}

Clothing pressure is significantly combined with the motion performance and pressure comfort such as sportswear for athletes. In previous studies, compression clothing was mainly concerned on physiological efficacy. Effect of partial pressure suits on heart rate, arterial pressure, cardiac output and ergonomics performance was discussed [1]. However, little attention has been focused on how to determine the most appropriate pressure in different body part to benefit physiological efficacy, while excessive pressure would affect heart and lung functions, and even perhaps resulted in serious damage to heath $[2,3]$. The above researches have clearly expounded the necessity of appropriate pressure. Consequently, before the proper pressure value of every part was determined, it was critical to grasp the law of pressure fluctuation of body parts completely during exercises. Previous researchers explored dynamic pressure of specified motions, and the specified motions were selected [4]. In fact, "dynamic pressure" in these studies was still static pressure of several fixed motions. The true dynamic pressure in continuous motion failed to be represented. Based on this, dynamic pressure of male lower limb wearing compression cycling shorts for continuous cycling motion was presented. A novel experimental approach to simulate dynamic pressure for continuous cycling motion was designed.

\section{Methodology}

\section{Subjects and compression cycling shorts}

Five healthy young cyclists were voluntarily participated in this study. All subjects were informed of the research purpose, and completed a health questionnaire. None of the subject was injury or pain. Six pairs of compression cycling shorts were manufactured by the same fabric with different negative ease allowance; serial numbers were $\mathrm{C}_{1}, \mathrm{C}_{2} \ldots$, and $\mathrm{C}_{6}$.

\section{Pressure measurement points}

Four measurement points were selected according to lower limbs muscle activity when cycling. In cycling rectus femoris, vastus lateralis, biceps femoris were the active muscles confirmed by skeletal muscle simulation. Considering muscle activation, middle of rectus femoris $\left(\mathrm{L}_{1}\right)$, middle medial thigh $\left(\mathrm{L}_{2}\right)$, middle lateral thigh $\left(\mathrm{L}_{3}\right)$, middle of biceps femoris $\left(\mathrm{L}_{4}\right)$ were selected as pressure measurement points, the location of every point was shown in Fig. 1. 


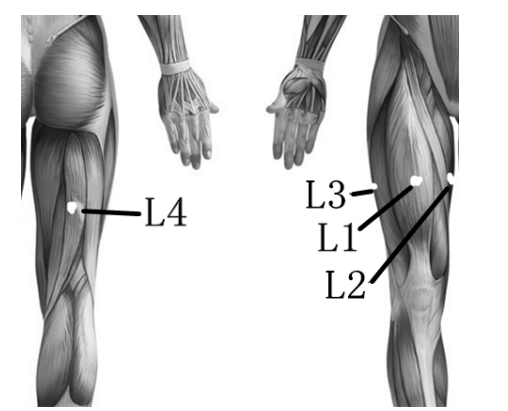

Fig. 1. Pressure measurement points.

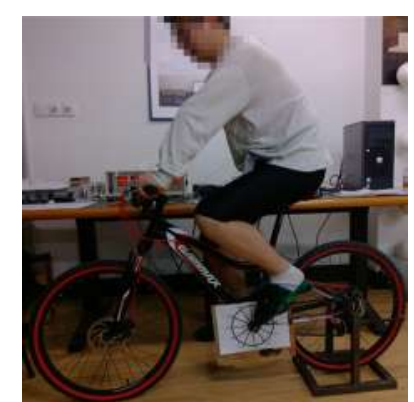

Fig. 2. Simulation of cycling.

\section{Simulation of cycling and skeletal muscle simulation}

Cycling in situ may simulate real scenario of cycling motion on flat. Experimental bicycle rear axle was supported from the ground with U-shaped bracket (see Fig. 2). An angle dial self-made was designed and fixed on the crank axle. The angle was divided into twelve, each $30^{\circ}$. So a periodic motion was disintegrated into twelve continuous motions. Then pressures of twelve motions were measured without interrupting. The human body modeling and simulation system was employed in simulating skeletal muscle activity rhythm. Three kinds of most active muscles (rectus femoris, biceps femoris, and vastus lateralis) were chosen and computed muscle stretch, the degree of muscle activation, and muscle force in the process of cycling.

\section{Pressure measurement protocol}

The experiment was performed as follows: Pressure value was measured with an air-pack type contact pressure measurement system. Before the beginning of experimental, saddle was adjusted to meet proper fit with different height and leg length [5]. Every subject was demanded 3 minutes of warm-up to adapt to test procedure. Sensors were accurately and firmly pasted at pressure measurement points and record pressure data of four points. Shorts were worn in order, and then pressure of standing still and dynamic pressure $\left(0^{\circ}, 30^{\circ} \ldots\right.$, and $\left.330^{\circ}\right)$ were measured continuously.

\section{Results and discussion}

\section{Middle rectus femoris muscle $\left(\mathrm{L}_{1}\right)$}

Pressure change was not significant from standing still to cycling posture at $\mathrm{L}_{1}$ (Fig. 3). Dynamic pressure first decreased and then increased gradually, the maximum at $0^{\circ}$ and the minimum at $120^{\circ} \sim 150^{\circ}$. Observing the result of skeletal muscle simulation in the process of cycling, the change of muscle stretch and dynamic pressure has a high consistency. Maximum stretch of rectus femoris muscle was found at $0^{\circ}$ and minimum at $120^{\circ} \sim 150^{\circ} .0^{\circ} \sim 120^{\circ}$ rectus femoris muscle gradually relaxed, corresponding the dynamic pressure value slowly decreased; in contrast, $150^{\circ} \sim 330^{\circ}$ dynamic pressure increased gradually as rectus femoris muscle contracted.

\section{Middle medial thigh $\left(\mathrm{L}_{2}\right)$}

The change trend of dynamic pressure at $\mathrm{L}_{1}$ and $\mathrm{L}_{2}$ were basically identical. Not only dynamic pressure corresponding same ease allowance shorts was lower compared with $\mathrm{L}_{2}$, but the change amplitude of dynamic pressure at $\mathrm{L}_{2}$ decreased about $50 \%$ by one-way ANOVA (Fig. 4). The result was related to shorts structure between sample shorts and thigh. Side seam was pulled to crotch. At $\mathrm{L}_{2}$ position, due to crotch stretch, sample shorts was not tightly fit with medial thigh in cycling posture when compared with $\mathrm{L}_{1}$ point. In this condition, pressure value measured would be smaller naturally and air-pack sensor was not sensitive to dynamic change. 


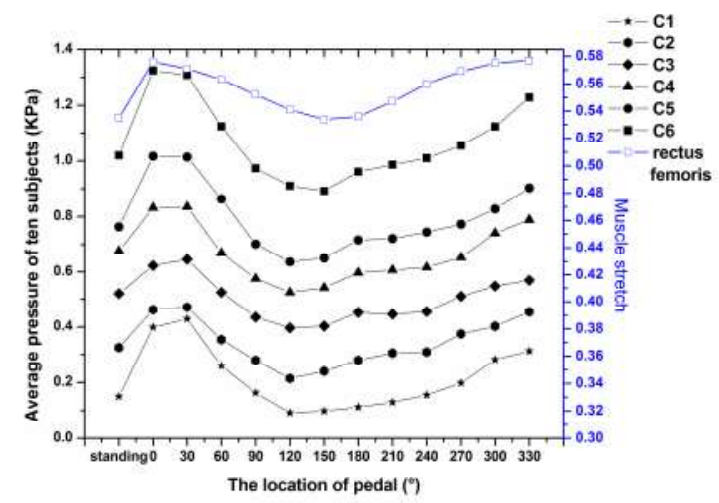

Fig. 3. Pressure change and muscle activation at $\mathrm{L}_{1}$.

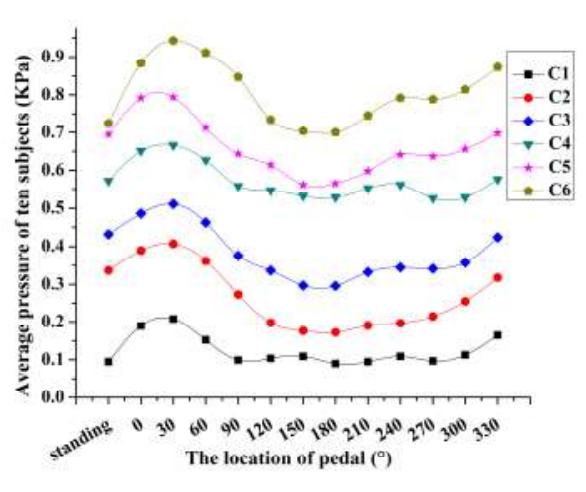

Fig. 4. Pressure change at $\mathrm{L}_{2}$.

\section{Middle lateral thigh $\left(\mathrm{L}_{3}\right)$}

Similarity of six curves reached more than $90 \%$ with correction analysis (Fig. 5). By ANOVA test, the result indicated that dynamic pressure of $\mathrm{L}_{3}$ had significant difference during cycling. Minimum of dynamic pressure for $\mathrm{L}_{3}$ occurs at $180^{\circ} \sim 210^{\circ}$, and vastus lateralis were relaxed state. Maximum existed at $0^{\circ} \sim 30^{\circ}$. The simulation of cycling motion showed muscle activity of lateral vatus lateralis was not obvious relatively. Furthermore, skin deformation of lateral thigh was smaller compared with middle anterior thigh. Dynamic pressure of $\mathrm{C}_{5}$ and $\mathrm{C}_{6}$ was much bigger than others. Consequently we believed that fabric properties did affect dynamic pressure.

\section{Middle biceps femoris $\left(\mathrm{L}_{4}\right)$}

Differences between maximum dynamic pressure and pressure of standing still for six sample shorts were $0.42 \sim 0.54 \mathrm{KPa}$ (Fig. 6). The maximum pressure still is at $0^{\circ}$. With one-way ANOVA test variances of dynamic pressure of six sample shorts were calculated. The result indicated that dynamic pressure had a little difference at middle biceps femoris. The maximum and minimum of dynamic pressure with biceps femoris of muscle stretch out of sync. But dynamic pressure and muscle stretch were basically the same change trend. The result revealed that muscle relaxation and contraction resolved pressure decrease and increase. The effect of muscle stretch to dynamic pressure change was also important.

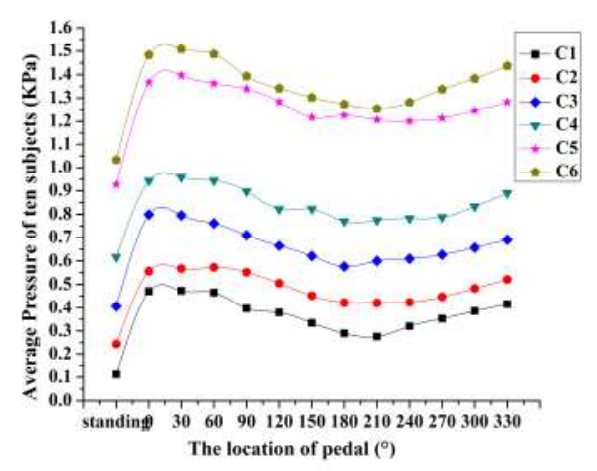

Fig. 5. Pressure change at $\mathrm{L}_{3}$. activation at $\mathrm{L}_{4}$.

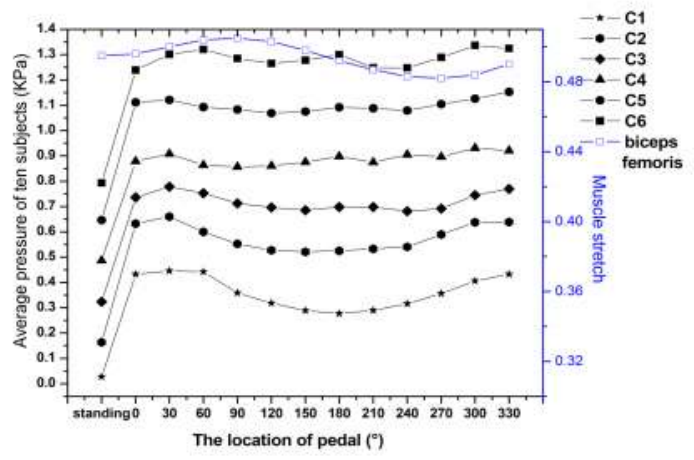

Fig. 6. Pressure change and muscle

The variation of dynamic pressure at eight points has been discussed in detail, respectively. Take $\mathrm{C}_{4}$ as example, other shorts were the similar result. Maximum pressure of all four points was at $0^{\circ} \sim 30^{\circ}$. Maximum pressure measured in experiment was $1.74 \mathrm{KPa}$. Previous research proposed that under low pressure (less than $4.65 \mathrm{KPa}$ ) external pressure accelerated skin blood flow of low limb [6]. On the base of this idea, in the certain pressure range shorts was the tighter the better, ease allowance of shorts still can be reduced continuously. From standing still to cycling posture, pressure of $\mathrm{L}_{3}$ and $\mathrm{L}_{4}$ increased highly, because skin deformation and curvature changed greatly at these two points. Variances can reflect fluctuation of dynamic pressure. According to the result of 
variances of four points, fluctuation of dynamic pressure was extreme at $\mathrm{L}_{1}$. Muscle activation of the point was most active. Subsequent cycling shorts design needs to highlight the point. Fluctuation of $\mathrm{L}_{4}$ was gentle, because muscle activation of $\mathrm{L}_{4}$ was very small. Muscle activation was significantly correlated with dynamic pressure from the analysis of $\mathrm{L}_{1}, \mathrm{~L}_{4}$. So far, similar results have not been found, other studies focused on the relationship of muscle activation and mechanical efficiency in cycling.

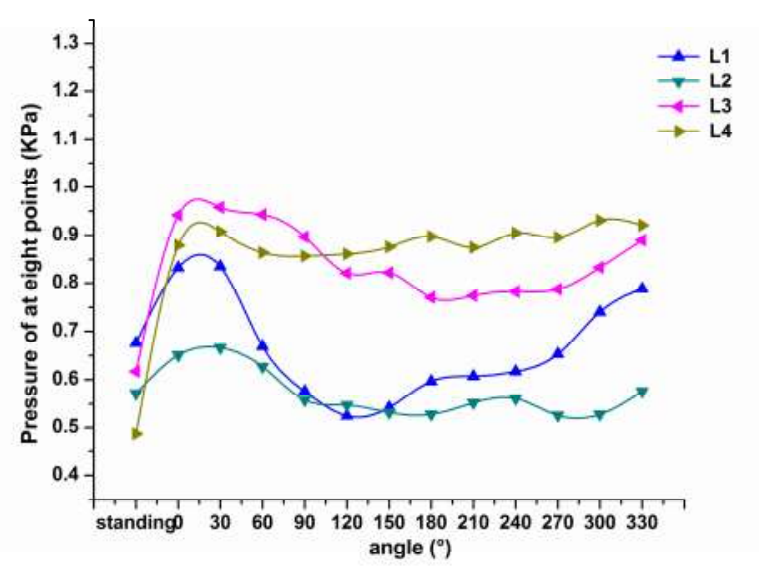

Fig. 7. Dynamic pressure of four points at $\mathrm{L}_{4}$.

\section{Conclusions}

A novel approach was developed to characterize dynamic pressure of male lower limb in cycling. The following results were founded. Pressure was dynamic change during cycling. The maximum dynamic pressure located at $0^{\circ} \sim 30^{\circ}$. Pressure gradually non-linear rose with ease allowance reducing. For middle rectus femoris $\left(\mathrm{L}_{1}\right)$, middle biceps femoris $\left(\mathrm{L}_{4}\right)$, dynamic pressure significantly associated with muscle activation. For cycling shorts at present, pressure was in the range of safety. Smaller ease allowance was recommended, but selecting the most appropriate pressure needed to be further investigated combining with the effect of pressure to function of human body in subsequent study.

\section{Acknowledgements}

The authors are grateful to all the volunteered participants who participated in this study.

\section{References}

[1] Yang, T., Ding, L., Zhang, C.G., Qin, Z.F., Hemodynamic simulation of partial pressure suits under pressure. J. Bj. Uni. Aeronautics and Astronautics, 39, pp. 1122-1126, 2013.

[2] Niwaya H., Evaluation technology of clothing comfortableness. J. Natl. Inst. Mater. Chem. Res, 7, pp. 269-282, 1999.

[3] Macintyre, L., Baird, M., Pressure garments for use in the treatment of hypertrophic scars-an evaluation of current construction techniques in NHS hospitals. Burns, 31, pp. 11-14, 2005.

[4] Zhang, X.H., Li, J., Wang, Y.Y., Dynamic pressure comfort of three garment sleeve structures. Proceedings of the Fiber Society 2009 Spring Conference pp, 1243-1248, 2009.

[5] Burke, E.R., Pruitt, A.L., High-tech cycling, in: Body positioning for cycling. Human Kinetics Publishers Inc., Champaign, pp. 72-92, 2003.

[6] Lu,Y.H., Dai, X.Q., Effect of External Pressure on Skin Blood Flow at Lower Limb in Different Postures. J. Fib. Bio. Info, 3, pp. 1159-1163, 2010. 